\title{
Coexister dans la communaute européenne : organisations religieuses et pregnance symbolique
}

Stefan Bratosin

\section{(2) OpenEdition}

1 Journals

Édition électronique

URL : http://journals.openedition.org/communicationorganisation/2887

DOI : 10.4000/communicationorganisation.2887

ISSN : $1775-3546$

Éditeur

Presses universitaires de Bordeaux

Édition imprimée

Date de publication : 1 mai 2004

ISSN : 1168-5549

Référence électronique

Stefan Bratosin, «Coexister dans la communaute européenne : organisations religieuses et pregnance symbolique », Communication et organisation [En ligne], 24 | 2004, mis en ligne le 27 mars 2012,

consulté le 20 avril 2019. URL : http://journals.openedition.org/communicationorganisation/2887 ;

DOI : 10.4000/communicationorganisation.2887

Ce document a été généré automatiquement le 20 avril 2019.

(c) Presses universitaires de Bordeaux 


\title{
Coexister dans la communaute européenne: organisations religieuses et pregnance symbolique
}

\author{
Stefan Bratosin
}

1 En réactivant les "jeux et les enjeux des frontières " ${ }^{1}$, le processus d'élargissement de l'Union européenne apporte, depuis peu au devant de la scène publique, la question politique de la coexistence des organisations religieuses dans l'espace européen. Les récentes prises de position dans le débat politique en faveur de l'héritage judéo-chrétien comme seul fond des repères symboliques capable de borner l'étendue européenne ${ }^{2}$, constituent l'une des meilleures illustrations, comme d'ailleurs, les dernières réitérations, par les décisions de Bruxelles, du clivage entre le monde occidental et le monde de l'Est ${ }^{3}$. Cependant, l'irruption publique du questionnement politique ne dévoile rien sur les conditions dans lesquelles se joue la coexistence des organisations religieuses en Europe. Elle les couvre uniquement et les renferme. Par conséquent, pour pouvoir saisir ces conditions, au lieu de s'attacher aux options politiques supposées conjoncturelles, il est convenable de considérer les regards religieux constitutifs des perspectives politiques, notamment ceux dont la contribution symbolique est particulièrement active dans le processus de construction européenne : l'optique orthodoxe de l'Est européen, la vision du catholicisme occidental et le mythe de l'œcuménisme.

\section{La sonorité nationaliste de la « symphonie » byzantine}

2 La perspective orthodoxe et la vision catholique occidentale participent d'une certaine convergence sur la « dimension spirituelle » de la communauté européenne - l'Europe est une création chrétienne -. convergence reprise déjà dans le discours politique ${ }^{4}$. Mais cette convergence d'angles de vue exploitée dans le discours politique cache des divergences extrêmement problématiques pour l'unité européenne. Tout en étant d'accord sur le christianisme comme agent déterminant de la construction européenne, 
les deux optiques attachent le point de départ de cette œuvre à des lieux symboliques différents - Byzance pour le christianisme oriental et Rome pour celui occidental correspondant à des fondements distincts en matière d'autorité organisationnelle: résultat du droit positif qui est conféré à une cité par les événements politiques, dans la perception orthodoxe, émanation de l'origine apostolique de la communauté, dans l'approche catholique.

3 Depuis la période constantinienne. au IV siècle, la tradition orthodoxe veut que l'organisation religieuse trouve son " équilibre » entre les deux institutions, spirituelle et temporelle, l'Église et l'État. Il s'agit d'une "symphonie »5byzantine dont les sons rassemblent trois registres historiques avec lesquels l'ecclésiologie orthodoxe contemporaine tisse les enjeux organisationnels de l'institution chrétienne. Ces trois registres sont l'autonomie, l'autocéphalie et la synodalité.

L'autonomie est un principe participant de l'ambiguïté récurrente d'une certaine indépendance organisationnelle vis-à-vis de l'État, tout en déployant une attitude de soumission à l'État, ce qui peut paraitre un paradoxe pour l'esprit occidental. Mais ceci ne l'est pas en vision orthodoxe puisque l'autonomie incarne «l'harmonie » entre l'État et l'Église, harmonie absolument nécessaire car elle est voulue par Dieu, tel que le montre l'épître de Paul aux Romains: "Tout homme doit se soumettre aux autorités qui gouvernent l'État, car il n'y a pas d'autorité qui ne vienne de Dieu " ${ }^{6}$. Se soumettre aux autorités civiles n'est donc pas une perte d'indépendance, car cette soumission n'est pas aux hommes, mais à la volonté divine. Ainsi dans cette perspective, puisque les deux institutions ont une origine commune, elles ne peuvent pas être soumises l'une à l'autre. La domination d'un pouvoir sur l'autre est inconcevable pour l'orthodoxie. La rivalité et l'opposition entre l'État et l'Église sont hétérodoxes. Les deux institutions sont distinctes, certes, mais complémentaires et par conséquent les deux pouvoirs qui ne sont ni séparés, ni en l'usions, sont appelés à se compléter et à collaborer. À la faveur de cette ambiguïté, l'organisation religieuse légitime, dans un sens, sa servitude à l'État, mais aussi, dans l'autre sens, l'instauration de n'importe quel régime politique. Par exemple, lors de l'instauration du régime communiste en Roumanie ${ }^{7}$, l'Église orthodoxe auparavant appui privilégie du pouvoir fasciste, parvient à se reconvertir sans problèmes particuliers en " servante » de la nouvelle société de travailleurs exigeant désormais à ses fidèles de se soumettre au nouveau régime car il n'est pas possible d'opposer les devoirs de citoyen et de croyant : «Opposer les devoirs religieux et civils ou les déclarer séparables. n'est pas une attitude approuvée par Dieu $»^{8}$. À son tour, le régime communiste avant recours au « roumanisme» - notion d'entre-deux-guerres désignant le nationalisme roumain -. a permis à l'organisation religieuse orthodoxe de s'inscrire dans une continuité culturelle, et par la même occasion dans la continuité de son idéologie pendant les régimes d'avant l'instauration $d u$ communisme. Plus exactement, en reprenant les thèmes du roumanisme. l'organisation religieuse orthodoxe s'est octroyé une légitimité par son rôle dans l'État et dans la nouvelle société.

Le deuxième registre de la «symphonie «byzantine qui relie le fonctionnement organisationnel à son inscription nationale est l'autocéphalie. Ce principe souligne la souveraineté et l'indépendance de l'organisation religieuse nationale par rapport au patriarcat œcuménique de Constantinople en toute matière sauf en ce qui concerne les dogmes. L'autocéphalie consacre le lien entre la nation et l'orthodoxie comme fait religieux. Dans certains cas, l'indépendance de l'Église est identifiée à l'indépendance même de l'État : «alors que l'Église s'était progressivement considérée comme le vecteur 
de l'identité ethnique et culturelle au cours du $19^{\mathrm{e}}$ siècle, la religion orthodoxe se trouve inextricablement liée, voire en fusion, avec le concept de nation et d'ethnie. Parallèlement à l'indépendance de l'État par rapport à la Porte ottomane. l'Église orthodoxe consacrait son indépendance vis-à-vis du patriarche de Constantinople soumis au pouvoir turc $»^{9}$. lin établissant le critère ethnique comme fondement de l'orthodoxie et de l'organisation de son institution, le principe d'autocéphalie constitue un lien entre l'identité, l'ethnicité et la confession à l'aide d'une argumentation nationaliste en ternies de "peuple né orthodoxe ». Ainsi l'usage orthodoxe du concept de nation en rapport avec l'organisation religieuse se révèle frappe, lui aussi d'une ambiguïté déconcertante, car la nation est entendue, selon les circonstances, soit dans son acception jacobine recouvrant l'ensemble des citoyens dans un État centralisé, soit dans le sens restrictif de l'ethnicité. Par le biais de l'autocéphalie, l'organisation religieuse orthodoxe se revendique en «symphonie » non seulement avec l'État, mais aussi avec la nation réduite le plus souvent, dans sa conception, au sens d'ethnie.

6 L'autonomie et l'autocéphalie organisationnelles orthodoxes pourraient poser, faute de lu synodalité, le problème d'unité institutionnelle. La synodalité est la caractéristique organisationnelle orthodoxe selon laquelle les décisions de principe pour l'unité de l'Église doivent être prises avec le consentement de l'ensemble des évêques sous la présidence du premier d'entre eux. Sur la synodalité repose la «symphonie » avec les Églises nationales sœurs. Ainsi, dans un contexte comme celui de la Roumanie ou de lu Bulgarie d'après la deuxième guerre mondiale, où l'organisation religieuse adhère à la démocratie populaire, le principe de synodalité sert pour légitimer la solidarité inter orthodoxe sous le régime communiste. L'unité de l'institution chrétienne est dans le monde orthodoxe le produit de la dimension synodale de son organisation et c'est grâce à ce type de fonctionnement que l'Église peut revendiquer dans cet espace son infaillibilité. Il s'agit d'une perspective diamétralement opposée à celle de l'infaillibilité catholique détenue par une seule personne, le pape.

7 En considérant ces trois registres de la «symphonie » byzantine, plusieurs situations apparaissent problématiques dans la perspective orthodoxe concernant le processus d'intégration et de construction de l'unité européenne. D'abord, l'intégration des organisations religieuses orthodoxes non seulement parmi les organisations religieuses de l'Union européenne, mais dans l'Union européenne tout simplement, soulève le problème posé par le lien entre l'ethnicité et autocéphalie promu par l'orthodoxie comme principe de fonctionnement social et politique : ce lien conduit au développement d'une idéologie d'exclusion et de discrimination. Selon cette idéologie, le citoyen d'un pays majoritairement orthodoxe et qui a la nationalité de ce pays, ne peut être envisagé autrement qu'orthodoxe. Autrement dit, l'habitant d'une autre nationalité ou non orthodoxe ne peut être un citoyen à part entière puisqu'il n'est pas affilié à l'histoire de l'ethnie. En second lieu, l'ethnicité, dans la conception orthodoxe, introduit une différence entre citoyenneté et nationalité : la citoyenneté est le fait de résider dans un pays, alors que la nationalité est directement liée à l'appartenance à une ethnie. Il en résulte une discrimination entre la nation ethnique majoritaire et les citoyens des minorités ethniques ou confessionnelles et cela en dépit des précautions constitutionnelles affichées comme vitrine pour une coexistence des organisations religieuses non problématique. Troisièmement, cette idéologie, en apparence tournée vers l'intérieur, est un véritable obstacle à l'extérieur pour l'intégration européenne des sociétés orthodoxes. Le modèle de société inspiré par la perspective orthodoxe étant 
foncièrement nationaliste, il cultive la crainte de l'unité européenne, car pour lui, cette unité exige une transformation spirituelle visant le renoncement à l'histoire nationale et à l'organisation religieuse sur des bases ethniques ${ }^{10}$ Le quatrième problème, sous-jacent au précédent, est la différence entre le concept de société développé à l'Est et celui mis en œuvre en Occident. Les orthodoxes considèrent que la construction européenne est tributaire de l'ecclésiologie catholique de la même manière que la nation orthodoxe est à l'image de l'ecclésiologie orthodoxe. Par conséquent, conformément à cette orientation idéologique, la nation orthodoxe participe à la culture européenne, certes, mais elle ne peut pas s'inscrire dans une construction européenne dont le fonctionnement relève du centralisme administratif, c'est-à-dire participant d'un processus de démotivation des spécificités nationales et ethniques intimement liées, dans l'optique orthodoxe, au danger à terme de l'effacement de sa spécificité spirituelle. Ainsi, dans la "symphonie » byzantine, la coexistence des organisations religieuses dans l'espace européen se joue comme juxtaposition de cloisonnements fonctionnels. Il s'agit d'un modèle de coexistence où l'enjeu n'est pas l'unité, mais l'harmonie européenne.

\section{Le discours occidental sur les universelles « racines » européennes}

8 À la symphonie byzantine faisant vibrer à l'Est les cordes des sensibilités nationales, correspond à l'Ouest, le discours du catholicisme latin sur les universelles «racines » européennes. Ce discours sur les «racines» chrétiennes de l'Europe, n'est pas uniquement la marque d'un moment culminant du processus d'inscription des organisations religieuses et tout particulièrement de l'Église catholique latine dans la construction politique et symbolique de l'Union européenne. Il est également l'expression d'une démarche constante de rappel publie croissant en puissance au sujet de la légitimité du rôle sociopolitique des organisations catholiques. Il s'agit d'une continuité entretenue par l'activité des organisations catholiques en rapport direct et permanent avec les différentes instances européennes ${ }^{11}$, mais aussi par des contacts et des échanges ponctuels fortement symboliques ${ }^{12}$ Conjointement à la démarche de rappel public de sa légitimité politique et sociale, l'église catholique développe aussi l'effort pour renégocier le statut du fait religieux afin de parvenir à une reconnaissance juridique avec des effets performatifs au niveau européen. Les deux facteurs déterminants de la conjoncture actuelle qui favorisent l'émergence des opportunités pour ouvrir une telle négociation sont d'une part le partage de plus en plus large de l'idée sociologique reprise dans le discours politique, selon laquelle la religion est un moyen pour stabiliser les rapports sociaux ${ }^{13}$ et d'autre part. le questionnement politique d'une Europe en quête de ses propres limites, de sa spécificité par rapport à ses voisins. Mais ce qui caractérise d'une manière décisive et interne le contenu « transactionnel » essentiel du discours catholique occidental sur les "racines" de l'Europe sont les deux thèmes fondamentaux et paradoxaux de la « culture commune » et des « valeurs universelles ».

Le thème d'une "culture commune » dans le discours du catholicisme occidental sur les « racines » chrétiennes de l'Europe est une sorte d'offre religieuse de sens à la demande politique en matière de définition et symbolisation d'une identité européenne. L'intérêt politique d'une telle offre consiste dans sa capacité de dépasser les délimitations sensibles instaurées par les identités nationales. Elle a l'aptitude apparente de contourner recueil d'une discussion sur une éventuelle nationalité européenne dont l'inconvénient majeur 
est la mise en cause des spécificités culturelles nationales. Cette offre est séduisante, car elle propose l'unité dans la diversité. Dans cette perspective l'organisation religieuse catholique a également l'avantage d'être bien plus à l'aise que les autres organisations religieuses ou politiques, car elle participe d'un fonctionnement qui se déploie depuis des siècles par-dessus des frontières géopolitiques. L'Église catholique apparaît ainsi comme détentrice de par son histoire et son expérience, d'un patrimoine commun, facilement convertible en "valeur culturelle commune » ou « héritage culturel commun ». L'idée du "patrimoine commun» européen est projetée à partir de deux angles de vues: l'un historique, l'autre anthropologique. Dans l'optique historique, le thème d'une culture commune sert d'argument pour déclencher et développer un travail de mémoire permettant aux différentes nations européennes de se rappeler que leur communauté spirituelle précède et constitue la base et le lien même de leur communauté économique. Cette œuvre de mémoire a trouvé un soutien direct dans le programme européen "Culture 2000 » qui finance les projets susceptibles de promouvoir "la connaissance mutuelle de l'histoire, des racines et des valeurs communes aux peuples de l'Europe, ainsi que de leur héritage culturel commun $»^{14}$. L'autre optique exploitée par l'Église catholique est la vision du monde et de l'homme, dont les leaders religieux en sont depuis toujours les apôtres. Cette vision inextricablement liée aux « racines «chrétiennes est appelée à produire les arguments capables d'élargir le rôle de l'organisation religieuse catholique du domaine privé au domaine publique afin d'apporter du sens au modèle, présumé unique, de société fondée sur des bases chrétienne : «les porteurs de sens, ceux qui essaient d'appeler à des considérations anthropologiques et éthiques comme fondamentales, ont quelques choses à dire à l'éclairage de la construction européenne, c'est-à-dire à un domaine public, et pas seulement au domaine privé des comportements personnels ${ }^{15}$ ».

Le thème d'une "culture commune " marque en dépit de son apparente fécondité, certaines limites pour la construction européenne. Il développe une

11 Association đEcuménique pour l'Église et Société. Cahier n 4/1997. p. 43, coexistence des organisations religieuse qui est paradoxalement en opposition avec l'idéal démocratique affiché par l'Union européenne. Pour parvenir à une identité commune, par le biais culturel de la communauté de religion, ce thème arrête les fouilles dans les vestiges culturels européens à l'époque où l'unité européenne était « assurée » par la symbolique du pouvoir pontifical. En termes de coexistence des organisations religieuses cela veut dire que l'Union européenne - dans la mesure où elle subit la séduction du discours catholique sur les "racines" - prenne le risque de succomber à un modèle d'unité européenne où la coexistence des organisations religieuses se joue non pas en termes de droits, mais de tolérances. Dans la même tendance de transmuer les droits en indulgence, la vision du monde et de l'homme proposée par l'Église catholique réactive un modèle de société vidé d'espace public et par conséquent d'opposition, un modèle de société qui abandonne tous les pouvoirs entre les mains d'une seule personne, ce qui équivaut à une condamnation de facto de la pluralité d'organisations religieuses, avec toutes les conséquences qui en découlent.

12 Le second thème développé par l'Église catholique dans son discours sur les "racines chrétiennes » de l'Europe est celui des "valeurs universelles ». Ce thème est en quelque sorte appelé par le besoin d'équilibre plutôt idéologique que discursif de l'ecclésiologie catholique, équilibre rendu instable par le thème de la « culture commune » européenne. En effet, en se présentant comme lieu de convergence symbolique d'une identité 
culturelle, en l'occurrence l'identité européenne. l'Église catholique a pris le risque de payer de sa propre identité qui par définition relève de l'universel. Le culturel s'attache toujours à un territoire. à des bornes et à des frontières, à des passages et à des discontinuités de l'espace. Or. sa spécificité la plus profonde et la plus chère est l'aptitude d'effacer toutes ces délimitations, son catholicisme, c'est-à-dire son caractère universel. Dans ee contexte, la récupération par l'Église catholique latine des valeurs essentielles dans la construction européenne comme "les droits de l'homme ", devient une nécessité à laquelle elle tache de satisfaire le plus souvent en aggravant les ambiguïtés. Ainsi. JeanPaul II s'emploie devant la Cour européenne des droits de l'homme à inclure, non pas en tant qu'expression de lois, mais en tant que valeur éthique, le concept de «droits de l'homme » dans une représentation. "le patrimoine commun », capable d'établir plus facilement un lien entre l'identité européenne et l'aspiration universelle visées par l'Église. Il affirme plus exactement que la notion de droits de l'homme «n'est pas simplement un catalogue de droits positifs, mais un ensemble de-valeurs sous-jacentes que la Convention appelle à juste titre le patrimoine commun d'idéaux et des principes des nations de l'Europe $»^{16}$. Mais, après une telle explication, le problème est davantage souligné. Ce qui est patrimoine universel n'est plus exclusivement européen et inversement, ce qui peut être qualifié comme « patrimoine commun » européen ne peut pas être universel. Plus exactement, si les droits de l'homme sont " patrimoine commun " européen, ils ne sont pas une valeur universelle ou alors ils sont une valeur universelle et par conséquent il est complètement antinomique de le revendiquer comme spécificité identitaire européenne.

Une autre voie pour satisfaire au besoin de souligner la participation de l'Église avec des valeurs universelles à la construction de l'identité européenne est celle de la mise en exergue de l'anthropologie judéo-chrétienne. Les droits de l'homme, par exemple, dans la vision du cardinal Poupard en qualité de président du Conseil Pontifical de la culture, sont l'apport du christianisme à la civilisation européenne parce que « l'idée des droits de l'homme (...) tire son origine de la conception chrétienne de l'homme comme personne unique, sujet de droits inaliénables, absolue dans sa dignité ${ }^{17}$ ». Cette voie relève alors une autre ambiguïté : soit les droits de l'homme concernent l'humanité entière et alors ils sont universels et non pas «tradition judéo-chrétienne », soit les droits de l'homme sont une tradition judéo-chrétienne et alors ils n'ont pas une valeur universelle vue que la tradition s'attache à une culture et donc à un territoire, d'où la discrimination comme résultat concret de l'application de ces droits.

Mais quelle que soit la voie empruntée pour indiquer le catholicisme comme origine commune de l'Europe ou quel que soit le degré d'ambiguïté dans un processus de réduction de l'universel à l'européen et d'élargissement de l'européen à l'universel, la coexistence des organisations religieuses proposée au travers du discours des universelles «racines» européennes se joue comme subordination inconditionnelle à l'Église catholique latine. Il s'agit d'un modèle de coexistence dont l'enjeu n'est pas l'unité, mais la crédibilité d'une pensée qui prône l'absence de matière pour une identité européenne hors du catholicisme occidental.

\section{Le mythe de l'œcuménisme}

15 Dans la mesure où l'Europe est considérée aussi bien à l'Est qu'à l'Ouest comme une construction d'origine chrétienne, un troisième lieu symbolique apparaît incontournable 
pour la mise en évidence des conditions de coexistence des organisations religieuses dans l'espace européen. Ce troisième lieu est celui de l'œcuménisme, c'est-à-dire celui du dialogue entre les organisations chrétiennes et qui ne doit pas être confondu avec les échanges inter religieuses. Au niveau européen, comme au niveau mondial, les parties en présence se regroupent autour de trois pôles distincts même si parfois frappés respectivement d'hétérogénéité : l'orthodoxie, le catholicisme et le protestantisme. Il faut noter que l'œcuménisme n'est pas une idéologie, mais une démarche qui consiste à donner à l'existence organisationnelle du christianisme une forme unitaire. Cette démarche a la fâcheuse caractéristique de recueillir en principe l'adhésion plus ou moins forte de l'ensemble des parties concernées et en même temps d'être rejetée dans sa traduction pratique par chacune de ces parties. Les raisons de cette situation paradoxale sont, certes, différentes selon les divers angles de vues, mais dans leur ensemble elles se livrent complémentairement. ce qui permet tout de même de saisir et d'expliciter des logiques de coexistence européenne développées par les organisations religieuses dans la perspective œcuménique.

16 Pendant les années où la guerre froide a scinde l'Europe politiquement, la démarche œcuménique a eu la tendance d'estomper la frontière historique entre la chrétienté de l'Est et celle de l'Occident. Le rassemblement œcuménique de Bâle en 1989 est peut-être l'un des derniers et sans doute le plus éloquent reflet de cette tendance. Cette rencontre organisée par la Conférence des Églises Européennes protestantes et orthodoxes (KEK) et le Conseil de vingt-cinq épiscopats catholiques européens (CCEE) a été coprésidée par le métropolite Alexis de Leningrad (à l'époque) et le cardinal Martini de Milan. Les délégations participantes réunissaient chacune des représentants orthodoxes, catholiques et protestants correspondant à la configuration religieuse des pays représentés. À cette occasion l'œcuménisme européen montre une certaine rupture avec la démarche "officielle» d'un dialogue affaire des hiérarchies des organisations religieuses et dévoile sa capacité d'occuper une place active dans les grands mouvements sociaux et civiques du moment: pacifisme, féminisme, écologie, lutte pour la justice internationale, droits de l'homme, non-violence etc. De plus, le caractère non doctrinal de ce rassemblement a permis d'espérer l'affranchissement de l'œuménisme de «l'autorité jumelée du Conseil pour l'Unité et de la Congrégation romaine pour la doctrine de la foi et aussi de se soustraire aux relations de type bureaucratique du Conseil CEcuménique des Églises (COE) $»^{18}$.

Mais la chute du mur de Berlin, quelque mois plus lard, après cette conférence de Bâle. a eu pour effet, d'une part, l'effacement des efforts précédents de jeter des ponts et d'ouvrir des voies praticables entre l'Est et l'Ouest chrétien de l'Europe et d'autre part, la réactivation des oppositions organisationnelles et structurelles attachées à des interprétations théologiques spécifiques des traditions et de l'histoire. Ainsi. 1997. à Graz, le second rassemblement œcuménique européen, en dépit d'une participation plus nombreuse, met en évidence le retour des prises de positions tranchantes et inflexibles. Revient, alors, dans le processus d'approchement entre les Églises orthodoxes et l'Église catholique, le problème de l'« uniatisme ", des Églises catholiques orientales unies, dites "Églises uniates». Ces organisations religieuses implantées en majorité dans l'Est européen entendant garder leur double spécificité - catholique et orientale - refusent la démarche œcuménique. En même temps les Églises orthodoxes interprètent la seule présence des Églises uniates comme une agression a la sensibilité religieuse nationale. Un tel écueil mine, dès lors, les échanges œcuméniques entre les Églises orthodoxes et 
l'Église catholique, faute de trouver une véritable solution à l'impasse de l'uniatisme. les deux formes d'organisations chrétiennes se sont retranchées dans la construction d'une symbolique pouvant renvoyer à une conception commune de leur coexistence. Les deux Églises conviennent de se dire "Églises sœurs». une façon d'indiquer un lien anthropologique et de réfuter toute idée de "rupture » avec les implications idéologiques et de reconnaissance d'autorité organisationnelle que cela comporte. Mais, cet " accord " sur la façon de se considérer mutuellement entre les Églises orthodoxes et l'Église catholique ne règle ni le problème de coexistence sur le même territoire des Églises uniates et des Églises orthodoxes, ni les divergences entre les Églises uniates et l'Église catholique occidentale. D'où l'appel de Jean Paul II à la réconciliation: "Les Églises orientales unies portent dans leur chair une terrible déchirure, car la pleine communion avec les Églises orientales orthodoxes, avec lesquelles elles partagent pourtant le patrimoine de leurs pères, ne peut être encore réalisée. Une conversion constante et commune est indispensable pour qu'elles procèdent avec résolution et élan en vue de la compréhension réciproque ${ }^{19}$. Et en même temps une sorte de mea culpa de l'Église catholique latine : « Une conversion est également exigée de la part de l'Église latine, afin qu'elle respecte et revalorise pleinement la dignité des Orientaux et qu'elle accueille avec gratitude les trésors spirituels que portent les Églises orientales catholiques au profit de la communion catholique tout entière ${ }^{20}$ »

Un deuxième nœud névralgique qui alimente les récits mythiques de l'œcuménisme est celui qui oppose les vues "traditionalistes » orthodoxes et les tendances «modernistes " de la théologie pratique protestante. Les tensions et les crises au sein du COL sont éloquentes. Les Églises orthodoxes qui manifestaient déjà depuis longtemps un certain mécontentement concernant l'engagement politique réclamé par des Églises occidentales, le déséquilibre du personnel administratif au COL. composé de trois cents fonctionnaires dont cinq seulement provenant des Églises membres orthodoxes etc.. menaçaient plus récemment de mettre lin à leur participation à cette instance œcuménique afin de marquer leur différence et leur opposition concernant le renouveau promu par les Églises protestantes sur des sujets comme les célébrations interconfessionnelles, le sacerdoce des femmes, la révision des normes éthiques qui règlent l'orientation sexuelle etc. Ainsi «à l'assemblée épiscopale russe de février 1997, les évêques ont affirmé que leur perplexité devant l'ordre du jour et les procédures du COL nécessitait une concertation inter orthodoxe sur l'ensemble des questions liées à la participation des Églises orthodoxes aux différents contacts avec le minute non orthodoxe ${ }^{21}$. Le retentissement de cette crise à l'intérieur de l'organisation orthodoxe est illustré de la manière la plus forte par le retrait de l'Église orthodoxe de Géorgie du COL et du KEK, afin d'éviter un schisme au sujet de l'œcuménisme. Cette illustration riche de significations est également l'expression des tensions qui traversent le monde des organisations orthodoxes, tensions dont l'impact sur la coexistence européenne des organisations religieuses porte aussi l'empreinte de la concurrence entre Moscou et Constantinople où le patriarcat subit actuellement une asphyxie en termes de politique turque, l'empreinte des différences culturelles entre Églises de tradition byzantine grecque et celles de tradition slave qui entravent les échanges panorthodoxe ou bien l'empreinte de l'incompatibilité d'humeur entre les autorités hiérarchiques des Églises impliquées dans des démarches institutionnelles au niveau européen et le clergé tourné vers la promotion des traditions d'une culture nationale. 
19 Plus récemment, la question qui se pose est de savoir si l'Église catholique va soutenir le problème soulevé par les Églises orthodoxes concernant la tendance d'ouverture théologique manifestée par les Églises protestantes. Mais rien n'est moins sûr. Sachant qu'au niveau européen cohabitent deux institutions principales - CCEE et KEK. - qui signifient au monde politique le positionnement symbolique de la coexistence actuelle des organisations chrétiennes dans l'espace européen, il convient d'observer que ce fonctionnement malgré son ambiguïté, constitue par son déploiement symbolique un facteur de stabilité. Les enjeux organisationnels. notamment de l'Église catholique, font qu'un statu quo est politiquement profitable pour l'Union européenne. Si, par exemple, l'Église catholique arrivait à s'imposer de facto comme seule organisation religieuse détentrice $d u$ "patrimoine culturel» européen, elle pourrait déclencher une crise aggravant son opposition historique avec les organisations orthodoxes et protestantes, constituant un camp irrémédiablement divisé peut-être sur le plan théologique, mais capable d'unité face à une «menace commune » concernant leurs rôles respectifs dans la construction spirituelle, sociale et politique de l'Europe. Dans un cas différent, si l'Église catholique se décide à faire de l'Église orthodoxe son allié privilégié, elle risque de laisser la voie libre aux seules organisations protestantes pour exploiter le patrimoine chrétien des « valeurs universelles » dans le processus de progrès social attaché à la construction européenne, un déséquilibre inconcevable dans l'acception catholique de l'Europe.

En somme, contrairement aux apparents objectifs originels, le modèle de coexistence des organisations religieuses proposé actuellement par l'œcuménisme en acte n'a pas pour enjeu l'unité européenne, mais l'équilibre, soit-il même précaire, d'un monde chrétien européen construit sans cesse à partir d'une logique de tensions inter organisationnelles.

\section{Conclusion}

Dans la perspective de l'Est européen, la coexistence des organisations religieuses doit faire face aux discours contraires aux valeurs démocratiques dont l'Occident se réclame le promoteur et le défenseur, alors que l'orthodoxie exploite ces mêmes valeurs auxquelles elle s'oppose. Contrairement, dans la perspective occidentale, la coexistence des organisations religieuses est confrontée aux discours d'un christianisme qui se revendique comme le dépositaire des "valeurs universelles" sur lesquelles repose immanquablement toute société démocratique, alors que le catholicisme fonctionne selon un modèle de pouvoir où l'universel dans son sens extensif, comme d'ailleurs dans son sens intensif également, ne peut avoir ni alternative, ni altérité. Dès lors, compter sur l'œcuménisme pour parvenir à l'unité spirituelle européenne est pratiquement non envisageable, même si l'œcuménisme, par son caractère mythique peut " faire marcher " ${ }^{22}$ la coexistence des organisations chrétiennes dans l'espace européen.

BIBLIOGRAPHIE

Droite et gauche françaises sont fortement divisées in Le Monde du 9/1 1/02. 
Exista o tendintâ de a distruge mostenirea erestinâ in Europa in Vestitorul Oriodoxiei du $1^{\mathrm{er}}$ juin 1999.

Il future dell'llnione in/. Avenire du 9 janvier 2003 Jean-Paul II reçoit M. Giscard d'Islamg in Zenith du 30 octobre 2002. Journal officiel des Communautés européennes du 28 octobre 1999 La Bible. Villiers-le-Bel Alliance biblique universelle. 1991.

L'Église est l'allié de tous ceux qui défendent les libertés véritables, discours devant la Cour européenne des droits de l'homme, in Documentation Catholique, le 6 novembre 1988

MM. Prodi. Strauss-kahn. Rocard Habermas au chevet de l'UE in Le Monde du 30 janvier 2003.

Religion et démocratie. Rapport du Conseil de l'Europe, Commission de la culture et de l'éducation, doc $n^{\circ} 8270$ du 27 novembre 1998

BRIA. L. Teologia fatâ in fatâ cu Biserica de azi in « Studii teologice ». $n^{\circ} 1 / 1990$. CAPELLE -

POGACEAN. A. Du « retour a l'orthodoxie » dans la Roumanie poste-communiste in L'Autre Europe. $\mathrm{n}^{\circ} 36-37 / 2002$

CERTEAU. M de. L'invention du quotidien Arts de faire. Paris : Gallimard. 1990. GILLET. ().. Religion el nationalisme L'idéologie de l'Église orthodoxe roumaine sous le régime communiste. Bruxelles Editions de l'ULB 1997 IONESCU, N. Indreptar ortodox, textes choisis de DC Amzar. Wiesbaden : I râpa ortodoxâ. 1957.

JEAN-PAUL II, Orientale lumen in Documentation Catholique, le 4 juin 1995 MARCU, G. Hristos si Cezarul sau Statul si Biserica in lumina Nouliu Testament in Mitropolia Ardealului nº 7-9/1977.

POUPARD. P. Aux fondements de la civilisation européenne in Christianisme et identité nationale Une certaine idée de l'Europe. Paris Bauchese. 1994 PRADEAU. C. Jeux et enjeux des frontières Talence PUB. 1994 VIGNON. J. Donner une âme a l'Europe in L'Europe lace aux défis. Réconciliation et sens. Association Æcuménique pour l'Église et Société. Cahier n 4 / 1997

\section{NOTES}

1. C. l'radeau. Jeux et enjeux des frontières. Talence. PUB. 1994.

2. Valéry Giseard d'Fstaing - dans la lignée de l'ancien chancelier Kohi qui voyait dans l'Union européenne un "club chrétien" - affirme en novembre 2002 que l'adhésion de la Turquie signifierait «la fin de l'Union européenne». François Bayrou réagit a ladite adhésion en rappelant que ce pays est « un pays musulman qui n'appartient pas a l'Europe » (Cf. Droite et gauche françaises sont fortement divisées in Le Monde du 9 novembre 20021 Romano Prodi met en place un groupe de travail sur la "dimension culturelle et spirituelle de l'Europe » qui ne comprend aucun représentant de l'islam (Cf. MM Prodi, Strauss-Kahn. Rocard. Habermas au chevet de l'UE in Le Monde du 30 janvier 2003) etc.

3. Les instances européennes, par exemple, ont décidé l'élargissement de l'Union Européenne aux pays où l'organisation religieuse majoritaire participe du christianisme occidental, mais elles ont refuse l'adhésion de la Roumanie et de la Bulgarie, pays majoritairement orthodoxes

4. Aux prises de position évoquées précédemment (Valéry Giscard d'I.slaing. François Bayrou. Romano Prodi) s'ajoutent Facilement d'autres aussi exphciles. connue celle, par exemple, de l'exambassadeur de Roumanie en France Alexandru Paleologu. qui affirmait que «l'Europe est une organisation appartenant aux chrétiens depuis des siècles » (Exista o tendintâ de a distruge mostenirea crestinâ in Europa in Vestitorul Ortodoxiei du $1^{\mathrm{er}}$ juin 1999.)

5. I Bria. Teologia fatà in fatâ cu Biserica de azi in Studü teologice, $n^{\circ} 1 / 1990$. p. 3.

6. Epître aux Romains 13 :1 (La Bible. Villiers-le-Bel, Alliance biblique universelle. 1991.) 
7. Le cas roumain constitue une référence particulièrement intéressante en raison de sa position au carrefour de différentes traditions - occidentales et orientales, latines, grecques et slaves mais aussi parce que son histoire a connu au cours du $2^{\mathrm{e}}$ siècle des régimes politiques aussi différents que le monarchisme, le fascisme, le communisme et le capitalisme (Voir, par exemple. A. Capelle-Pogacean. Du « retour à l'orthodoxie » dans la Roumanie post-communiste in L'Autre Europe. $\mathrm{n}^{\circ}$ 36-37 / 2002. p. 117-139; O. Gillet. Religion et nationalisme l'idéologie de l'Eglise orthodoxe roumaine sous le régime communiste. Bruxelles. Editions de l'ULB. 1997.)

8. G Marcu. Hristos si Cezarul sau Statut si Biserica in lumina Noului Testament in Mitropolia Ardealului ${ }^{\circ}$ 7-9/1977. p. 492

9. O. Gillet, E cclésiologie orthodoxe et nationalisme en Roumanie, en ligne. http:// european.memorv.free.fr/textes/roumanie. dernière visite le 12/05/0.3

10. Voir, par exemple. $\mathrm{N}$ lonescu. Indreptar ortodox. textes choisis de D.C. Amzar. Wiesbaden. Faitia ortodoxâ 1957. p. 83

11. Le dialogue institutionnel avec la Commission européenne, la présence des ordres religieux et des ONG confessionnelles dans les actions communautaires européennes, la mise en place des structures transnationales mobilisant le laïcat européen etc.

12. Par exemple, l'audience du président du Parlement européen au Vatican, au début du mois de janvier 200.3. audience au cours de laquelle Patrick Cox $a^{12}$ Par exemple, l'audience du président du Parlement européen au Vatican, au début du mois de janvier 2003. audience au cours de laquelle Patrick Cox a invité le pape à Strasbourg pour «secouer les consciences » des députés et « rappeler son message à propos de l'identité chrétienne de l'Europe » (Cf. Il future dell'Unione in L'Avvenire du 9 janvier 2003). ou l'assurance donnée en 2002 par Valéry Giscard d'Estaing au Vatican concernant l'inscription de l'« héritage religieux » dans la constitution européenne (Cf. Jean-Paul II reçoit M Giscard d'Estaing in Zénith du.30 octobre 2002).

13. Cf. Religion et démocratie. Rapport du Conseil de l'Europe. Commission de la culture et de l'éducation, doc. $\mathrm{n}^{\circ} 8270$ du 27 novembre 1998.

14. Journal officiel des Communautés européennes du 28 octobre 1999.

15. J Vignon. Donner une âme a l'Europe in L'Europe lace aux défis Réconciliation et sens.

16. 1 'Eglise est l'allié de tous ceux qui détendent les libertés véritables, discours devant la Cour européenne des droits de l'homme, in Documentation Catholique, 6 novembre 1988. p. 1004-1005

17. P. Poupard. Aux fondement de la civilisation européenne in Christianisme et identité nationale Une certaine idée de l'Europe. Paris : Bauchese 1994. p. 11

18. J. Lirootaers. Un quart de siècle d'œecuménisme, en ligne http://www ksurf net/stiean/art 15. dernière visite le 12/05/03

19. Jean-Paul II. (orientale lumen in Documentation Catholique, le 4 juin 1995. p. 527

20. Jean-Paul II. op cit

21. J Grootaers. op cit

22. M. de Certeau. L'invention du quotidien. Arts de faire, Paris. Gallimard. 1990. p. 154.

\section{AUTEUR}

\section{STEFAN BRATOSIN}

IUT de Tarbes, Université de Toulouse 3/LERASS 